\title{
Dual Monitoring of Surface Reactions in Real Time by Combined Surface-Plasmon Resonance and Field-Effect Transistor Interrogation
}

Patrik Aspermair, ${ }^{\perp}$ Ulrich Ramach, ${ }^{\perp}$ Ciril Reiner-Rozman, Stefan Fossati, Bernadette Lechner, Sergio E. Moya, Omar Azzaroni, Jakub Dostalek, Sabine Szunerits, Wolfgang Knoll, and Johannes Bintinger*

Cite This: J. Am. Chem. Soc. 2020, 142, 11709-11716

Read Online

ABSTRACT: By combining surface plasmon resonance (SPR) and electrolyte gated field-effect transistor (EG-FET) methods in a single analytical device we introduce a novel tool for surface investigations, enabling simultaneous measurements of the surface mass and charge density changes in real time. This is realized using a gold sensor surface that simultaneously serves as a gate electrode of the EG-FET and as the SPR active interface. This novel platform has the potential to provide new insights into (bio)adsorption processes on planar solid surfaces by directly relating complementary measurement principles based on (i) detuning of SPR as a result of the modification of the interfacial refractive index profile by surface adsorption processes and (ii) change of output current

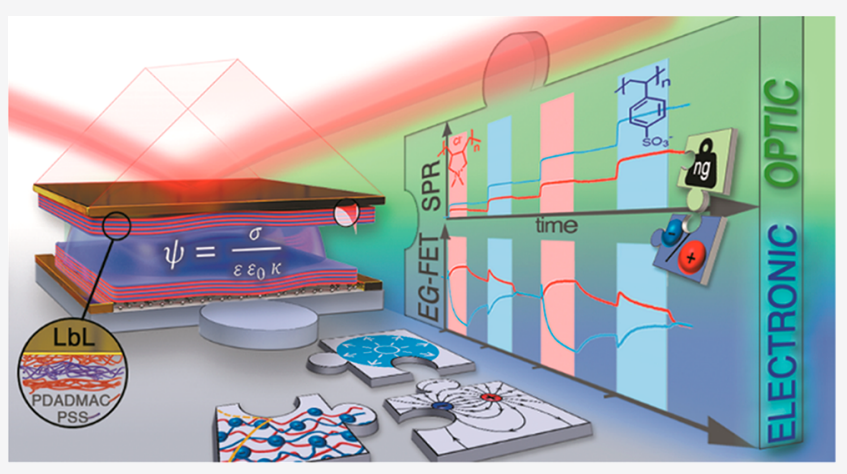
as a result of the emanating effective gate voltage modulations.

Furthermore, combination of the two complementary sensing concepts allows for the comparison and respective validation of both analytical techniques. A theoretical model is derived describing the mass uptake and evolution of surface charge density during polyelectrolyte multilayer formation. We demonstrate the potential of this combined platform through the observation of layer-bylayer assembly of PDADMAC and PSS. These simultaneous label-free and real-time measurements allow new insights into complex processes at the solid-liquid interface (like non-Fickian ion diffusion), which are beyond the scope of each individual tool.

\section{INTRODUCTION}

Electronic sensing devices including those based on electrolyte gated field-effect transistors (EG-FETs) have attracted increasing attention in recent years due to their potential for the use in compact and cost-efficient analytical devices. ${ }^{1-7}$ Despite the progress in understanding the underlying principles and even demonstrating label-free single-molecule detection, ${ }^{8}$ no commercial EG-FET biosensor has yet entered the market. Specifically, low reproducibility, unspecific binding, sensor drift, and batch to batch variations have hindered largescale deployment of this emerging class of biosensors. ${ }^{9}$ In the early 1980s and 1990s, comparable challenges were addressed in the field of surface plasmon resonance (SPR) biosensor systems. ${ }^{10,11}$ Research carried out over the last decades has paved the way for establishing this method in the market of biomolecular interaction analysis, ${ }^{12}$ and we have witnessed the gradual advancement of this technology for rapid detection of chemical and biological species. ${ }^{10,13}$ SPR biosensors allow direct label-free monitoring of molecular affinity binding events on the sensor surface associated with changes in surface mass density. ${ }^{14,15}$ They are probed by the confined optical field of surface plasmons and monitored through variations in the local refractive index. Over the last years, progress in the instrumentation of SPR biosensor technology has allowed for detection of minute changes in surface mass density, enabling analysis of molecules with low molecular weight and species that are present in trace amounts in analyzed liquid samples. However, they typically rely on complex optical systems that are deployed in specialized laboratories, particularly when combined with other techniques for measurement of additional parameters beyond the affinity binding rates. These parameters include identification of biomolecular interactions by coupling SPR biosensors with mass spectrometry, ${ }^{16}$ surface-enhanced Raman spectrometry, ${ }^{17}$ fluorescence spectroscopy, ${ }^{18}$ or monitoring of conformational changes of biomolecules with

Received: November 11, 2019

Published: May 14, 2020 
plasmonically enhanced IR spectroscopy. ${ }^{19}$ In comparison with these methods, EG-FET systems offer the advantage of simpler device architecture combined with an electronic readout principle, scalable cost-efficient production, low power consumption, and facile integration into point-of-care platforms that do not require specialists for operation. ${ }^{7}$ The measurement principle is based on sensing of complementary changes caused by electric field effects ${ }^{2,20-25}$ associated with changes in charge distribution upon capture of a target species. This approach allows probing at closer proximity to the sensor surface $^{26}$ than SPR and has the potential to monitor effects that are beyond the scope of the optical SPR technique, for instance, conformational changes of biomolecule surface reactions. $^{27}$ As many biologically relevant processes are inherently linked to mass and charge variations, fusing different sensing techniques into one multifunctional instrument could offer intriguing possibilities to investigate phenomena from different perspectives. Only a few reports in this context attempt to separate mass and charge effects, ${ }^{28-30}$ and neither offered temporal resolution, performed proper spatial coupling of the system, nor provided a satisfying theoretical framework.

Here, we report an approach to combine SPR (optical) and EG-FET (electronic) readouts for simultaneous and real-time observation of optical and electronic aspects of molecular interactions. It is realized that using a gold sensor surface simultaneously serves as the gate electrode of the EG-FET and as the SPR-active interface (Figure 1). We demonstrate the

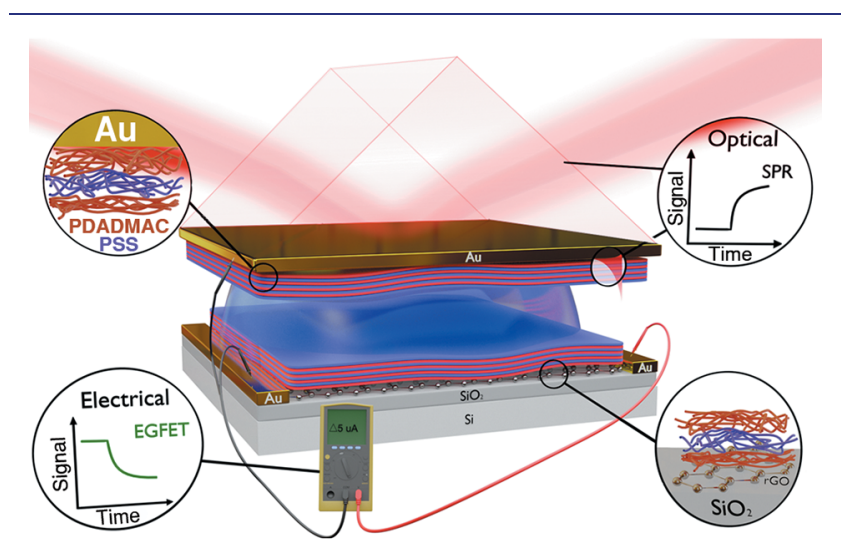

Figure 1. Schematic illustration of the combined SPR/EG-FET setup. Top Au electrode plays the dual role of SPR sensing surface and EGFET gate electrode.

capabilities of the developed SPR/EG-FET platform through the real-time observation of layer-by-layer (LbL) assembly of charged polyelectrolytes. We elucidate surface effects including intralayer ion diffusion processes from complementary techniques. This surface architecture represents a wellestablished system that offers a simple bottom-up modification strategy on different substrates. ${ }^{31-35} \mathrm{LbL}$ multilayers are sequentially assembled using the attractive forces between oppositely charged polyelectrolytes and typically characterized after each layer deposition step. Moreover, this technology shows great potential in multiple, diverse fields such as fuel cells, $^{36}$ batteries, ${ }^{37}$ drug delivery, ${ }^{38}$ and water desalination, ${ }^{39}$ but a better understanding of the intrinsic processes and the resulting film properties is essential for guiding the development of new films and specific applications. ${ }^{40,41}$

Real-time measurement using the SPR/EG-FET approach offer a means to observe typically inaccessible effects associated with the kinetics of binding and redistribution of mass and charge density during the growth of individual layers. In addition, the reported approach of the bifunctional sensor can be extended in a straightforward manner for measurement of other (bio)molecular interactions and serve to further develop these platforms and to elucidate surface effects that neither SPR nor EG-FET can address individually. Due to their relatively large mass and high charge density, LbL architectures are well-suited model systems to demonstrate the capability of the novel platform for monitoring mass deposition and charge distribution.

\section{RESULTS}

SPR chemo-optical signal transduction relies on the detuning of the resonant optical excitation of surface plasmons on a metallic surface. It occurs due to the increase of refractive index upon growth of a (bio)molecular assembly on the sensor surface. ${ }^{11}$ The observed detuning of SPR can be converted into changes in the surface mass density $\Gamma$ of the (bio)molecular layer using effective medium theory. ${ }^{42,43}$ In contrast, the chemo-electrical signal transduction in EG-FETs is based on the locally induced electric field variations induced by surface charge density changes on the sensor modulating the Fermi level, ${ }^{44}$ observed as a shift of the Dirac point $V_{i}$, the voltage of lowest conductance (Figure 2). We observed LbL assembly of polyelectrolyte multilayers (PEM) using SPR with Kretschmann configuration of the attenuated total internal reflection method.

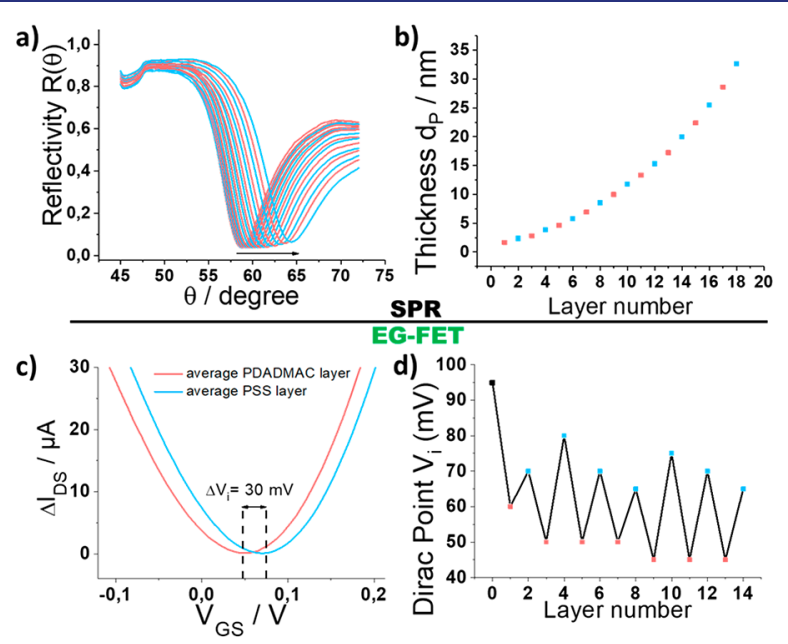

Figure 2. (Top) Sequential LbL assembly monitored by a SPR system using a planar gold surface. (a) Shift of the resonance angle as result of layer deposition, and (b) total thickness of the adsorbed films as a function of deposited layers. (Bottom) LbL assembly monitored in situ by an EG-FET system based on rGO. (c) Transfer curves of EGFET for PDADMAC/PSS assemblies, and (d) change of the Dirac points $V_{i}$ as a function of the number of adsorbed layers and their respective charges.

The resonant excitation of surface plasmons manifests itself as a dip in the angular reflectivity $R(\theta)$ (Figure 2a). At the resonant angle, the excited surface plasmons probe the gold sensor surface with a PEM that was sequentially grown from positively charged poly(diallyldimethylammonium chloride) (PDADMAC; average molecular weight $<100 \mathrm{kDa}$ ) and negatively charged poly(sodium 4-styrenesulfonate) (PSS; average molecular weight approximately $70 \mathrm{kDa}$ ) during 
continuous flow $(100 \mu \mathrm{L} / \mathrm{min}$,). Polymer solutions $(1 \mathrm{mg} / \mathrm{mL}$ in $0.1 \mathrm{M} \mathrm{KCl}$ aqueous solution) with refractive index $n_{\mathrm{s}}=1.333$ were sequentially pumped over the UV-ozone activated gold sensor surface with intermediate rinsing steps (see Supporting Information for details). After growth of each layer, angular reflectivity scans $R(\theta)$ were recorded and the thickness $d_{\mathrm{p}}$ and refractive index $n_{\mathrm{p}}$ of the assembled PEM were obtained by fitting the spectra using the Fresnel reflectivity model. Analysis was described in detail in previous work, ${ }^{35}$ and the refractive index of PDADMAC/PSS layers was measured as $n_{\mathrm{p}}=1.577$ when dried in contact with air. After swelling of the PEM in $0.1 \mathrm{M}$ aqueous $\mathrm{KCl}$ solution, its refractive decreases to $n_{\mathrm{p}}=1.476$. Fitting $R(\theta)$ for swollen PEMs allows determining the dependence of layer thickness $d_{\mathrm{p}}$ on the number of growth steps, which can be seen in Figure $2 b$ (corresponding kinetic measurements as well as angular scans are shown in Figure S1). In accordance with previous reports, ${ }^{45}$ this dependence exhibits a parabolic trend, and the average increase of $\Delta d_{\mathrm{p}}=0.95 \mathrm{~nm}$ was determined for the first eight layers. The average surface mass density increase $\Delta \Gamma$ per layer associated with LbL deposition of the PEM was determined by ${ }^{46,47}$

$$
\Delta \Gamma=\Delta d_{\mathrm{p}} \cdot\left(n_{\mathrm{p}}-n_{\mathrm{s}}\right) \cdot\left(\frac{\partial n}{\partial c}\right)^{-1}
$$

resulting in $\Delta \Gamma=69 \mathrm{ng} / \mathrm{cm}^{2}$. In this equation the coefficient $\partial n / \partial c=0.2 \mathrm{~mm}^{3} \mathrm{mg}^{-1}$ relates the changes in refractive index with the concentration of (bio)polymers bound to the surface. $^{48}$

We further investigated the PEM assembly using reducedgraphene oxide-based field-effect transistors (rGO-FET) by monitoring the resulting Dirac point shifts $\Delta V_{i}$ (Figure $2 \mathrm{c}$ and 2d). ${ }^{34,35}$ rGO-FETs were fabricated by previously reported procedures (see Supporting Information). ${ }^{35}$ All measurements were performed in a dedicated flow cell (Figures S19 and S20). Electrical measurements were performed by applying a $50 \mathrm{mV}$ source-drain bias and sweeping the gate potential $V_{\mathrm{GS}}$ while monitoring the current between the drain and the source $I_{\mathrm{DS}}$ using a Keysight U2722A instrument with custom-made LabVIEW software (Figure S21). An $\mathrm{Ag} / \mathrm{AgCl}$ reference electrode was used as a gate electrode. As shown in Figure 2c, positively charged PDADMAC layers shift the transfer curve $\left(I_{\mathrm{DS}} V_{\mathrm{GS}}\right)$ to more negative voltage values compared to a positive Dirac shift $\Delta V_{i}$ introduced by negatively charged PSS layers, which is in agreement with previous publications. ${ }^{49}$ The observed $\Delta V_{i}$ between alternating layers are on the order of $25-40 \mathrm{mV}$. Furthermore, the dependence on the ionic strength of the $\mathrm{KCl}$ solutions was also evaluated (Figure S11).

Interestingly, these shifts obtained from dynamic LbLprocesses are smaller than values obtained from static assembly processes, as recently shown by our group, ${ }^{35}$ and can be explained by the absent drying step after each layer deposition. The drying step causes a collapse of the polymer layer and thus increases the surface charge density $\sigma$.

After determining the viability of in situ monitoring $\mathrm{LbL}$ assembly using individual SPR and EG-FET measurements, each in a separate flow cell configuration, the concept of the dual-electro/plasmonic signal transduction was demonstrated with the combined SPR/EG-FET tool. A $50 \mathrm{~nm}$ thin gold layer used in SPR measurements with Kretschmann configuration was simultaneously employed as the gate electrode of the EG-
FET. A custom-made polydimethylsiloxane gasket defining the flow cell volume ( $5 \mu \mathrm{L}, 400 \mu \mathrm{m}$ flow channel thickness) attached the EG-FET to the SPR substrate. The flow cell was sealed using a 3D-printed holder with a commercially available interdigitated electrode chip (Micrux IDE1) using rGO as channel material (Figures 1, S17, and S19).

Sequential LbL growth of PEM, composed of PDADMAC and PSS, was monitored in parallel using the optical (SPR) and electronic (EG-FET) readout channels. The acquired sensor response kinetics are presented in Figure 3 for the growth of layers 3-8, revealing a stepwise increase in the SPR response and an alternating, more complex, electrical EG-FET signal.
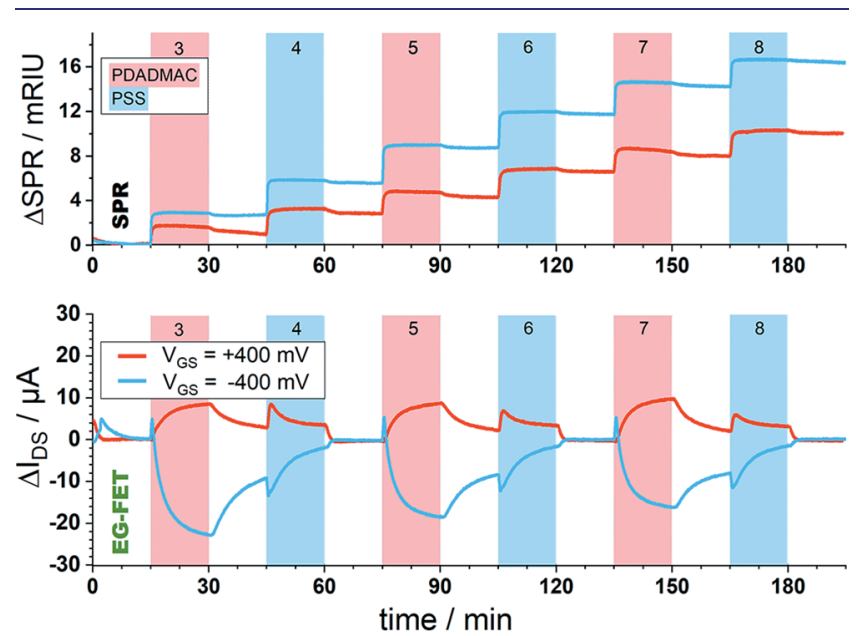

Figure 3. In situ readout of the sequential growth of alternating PDADMAC (red bars, $N=3,5,7$ ) and PSS (blue bars, $N=4,6,8$ ) layers by the use of SPR (upper graph) and EG-FET (bottom graph). Device was operated with applied negative (blue curve) and positive (red curve) $V_{\mathrm{GS}}$ voltages. Baseline correction was applied to level post-PSS $I_{\mathrm{DS}}$ current values to zero. See Figures $S 1$ and $S 13$ for raw data and gate leakage current values.

The first two layers (see Figure S1 for raw data) act as precursor and ensure sufficient PEM coverage for the subsequent layers. ${ }^{50}$ The SPR sensor response was measured in refractive index unit (RIU) by calibrating the sensor to bulk refractive index changes $n_{\mathrm{s}}$ (Figure S1). Prior to growth of the second pair of PDADMAC/PSS layers (layers 3 and 4), a baseline was established ( $\mathrm{KCl}, 100 \mathrm{mM}, 0-15 \mathrm{~min})$. Then the solution with positively charged PDADMAC was injected (15 $\mathrm{min})$, and a rapid increase in SPR response was observed as a result of surface mass deposition. The system was rinsed with $\mathrm{KCl}$ for $15 \mathrm{~min}$, and a rapid small decrease in the SPR signal was observed due to the bulk refractive index change $n_{\mathrm{s}}$ and the desorption of loosely bound polymer chains. ${ }^{51}$ Next, the solution with negatively charged PSS was injected for $15 \mathrm{~min}$ followed again by a $15 \mathrm{~min}$ rinsing step, leading to a similar increase in SPR signal. The growth of the first 8 layers was linearly approximated (Figure S12) and shows a gatevoltage dependency.

The equilibrium $\Delta$ SPR signal of 10-16 mRIU (and respective surface mass density $\Delta \Gamma$ and PEM thickness $d_{\mathrm{p}}$ ) are in accordance with those measured in static mode and presented in Figure 2. Interestingly, the overall surface mass density $\Gamma$ of the grown PEM was about $60 \%$ higher for the negative $V_{\mathrm{GS}}$ applied to the gold surface compared to the positive one due to the respective changes in the surface mass density of the initial positively charged PDADMAC layer. Due 
to the polarizability of charged polymers, the polymer chains exhibit instantaneous response to the electrostatic attraction by $V_{\mathrm{Gs}}{ }^{52}$ Hence, electrophoretic deposition occurs and the electrostatic compensation process between cationic and anionic polyelectrolytes is stronger, resulting in thicker films as seen by the SPR signals (Figure S12). This corresponds to a mean layer growth of $d_{\mathrm{p}}=2.82 \pm 0.06 \mathrm{~nm}$ with $\Delta \Gamma=202 \pm$ $4 \mathrm{ng} / \mathrm{cm}^{2}$ for $V_{\mathrm{GS}}=-400 \mathrm{mV}$ and $d_{\mathrm{p}}=1.69 \pm 0.06 \mathrm{~nm}$ with $\Delta \Gamma=121 \pm 4 \mathrm{ng} / \mathrm{cm}^{2}$ for $V_{\mathrm{GS}}=+400 \mathrm{mV}$, as extracted from the coupled SPR/EG-FET system according to eq 1 . As the same PEM is formed on both interfaces of the flow cell, we further assume that both SPR and EG-FET channels respond to the same supramolecular architecture. While the SPR is sensitive to the binding of higher molecular weight PDADMAC and PSS polymers, it will not respond to the presence of low molecular weight $\mathrm{K}^{+}$and $\mathrm{Cl}^{-}$ions. In contrast, the EG-FET responds to charge density variations independent of the molecular weight.

In comparison to the SPR, the response observed with the EG-FET channel $\left(\Delta I_{\mathrm{DS}}\right)$, shown in Figure 3, shows different behavior. In this experiment changes in $\Delta I_{\mathrm{DS}}$ were measured in time for a fixed applied gate-potential $\left(V_{\mathrm{GS}}\right)$, which defines the working point of the EG-FET system. The measured current changes are proportional to the slope of the $I_{\mathrm{DS}} V_{\mathrm{GS}}$ curve, which is opposite for the set $V_{\mathrm{GS}}= \pm 400 \mathrm{mV}$ (Figure S14). The binding of positively charged PDADMAC polymer and negatively charged PSS polymer is accompanied by opposite changes in $I_{\mathrm{DS}}$ current as the binding of these polymers shifts the Dirac point $V_{i}$ to more negative or positive voltages, respectively (Figure $2 \mathrm{c}$ ). Only the ambipolar properties of certain semiconducting materials (such as rGO) allow for using both positive and negative gate voltages $\left(V_{\mathrm{GS}}\right)$ and thus investigating the electric field dependence of the LbL adsorption.

Strikingly, trends in the EG-FET signal due to growth of PEMs are inherently different from those observed with SPR, in terms of both magnitude and kinetics. For example, deposition of positively charged PDADMAC gradually increases the $I_{\mathrm{DS}}$ current (in the case of $V_{\mathrm{GS}}=+400 \mathrm{mV}$ ) until equilibrium is reached in about $15 \mathrm{~min}$, thus taking 30 times longer than the SPR signal. At the beginning of PSS injection, a rapid increase in $I_{\mathrm{DS}}$ (in the case of $V_{\mathrm{GS}}=+400$ $\mathrm{mV}$ ) occurs in about $1 \mathrm{~min}$ and overlays with a slow competing decrease that reaches equilibrium in about $15 \mathrm{~min}$. As expected, the current output sign is reversed when applying a negative gate potential $\left(V_{\mathrm{GS}}=-400 \mathrm{mV}\right)$.

A detailed investigation of the EG-FET signal reveals two contributions, both exhibiting exponential behavior with different time constants (Figure S2). The two processes can be attributed to the accumulated charge density $\sigma$ (EG-FET), which is composed of (i) $\sigma_{a}$, originating from adsorbed charged mass density (SPR), and (ii) $\sigma_{\mathrm{i}}$, induced by capacitive effects, according to $\sigma=\sigma_{\mathrm{a}}+\sigma_{\mathrm{i}}$.

Changes of the surface charge density correspond to variations of the surface potential $\psi$ (Debye-Hückel, eq 2), thus modifying $I_{\mathrm{DS}}$

$$
\psi=\frac{\sigma}{\varepsilon \varepsilon_{0} \kappa}
$$

where $\kappa=1 / \lambda_{\mathrm{D}}=\left[\left(2 z^{2} e^{2} n_{0}\right) /\left(\varepsilon k_{\mathrm{B}} T\right)\right]^{-1 / 2}$, with Debye length $\lambda_{\mathrm{D}}$, ion valency $z$, electron charge $e$, ion concentration of the bulk $n_{0}$, Boltzmann constant $k_{\mathrm{B}}$, temperature $T$, permittivity constant $\varepsilon_{0}$, and relative permittivity $\varepsilon$ of the solution.
Experimental confirmation was obtained by eliminating any capacitive contributions $\left(\sigma_{\mathrm{i}}=0\right)$, which was realized by replacing the gate electrode with a nonconductive substrate (Figure S4), thus leading to $\sigma=\sigma_{\mathrm{a}}$. Such two-terminal devices have been demonstrated for sensing applications. ${ }^{53}$ As shown in Figure 4, the time constants $t_{0}$ for noncapacitive experiments

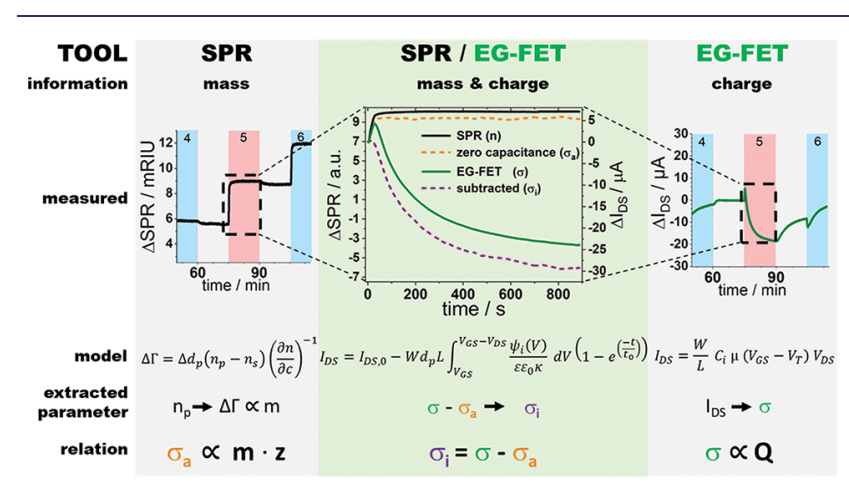

Figure 4. (Left and right) Detailed signal response of layer 5 from Figure 3. (Middle) Obtained capacitance contribution by subtracting the zero-capacitance measurement from the EG-FET response upon layer formation.

(orange dashed line) are nearly identical to the SPR response (black solid line), which is attributed to the adsorbed surface charge density $\sigma_{\mathrm{a}}$. Hence, we demonstrated that the SPR mass uptake is proportional to $\sigma_{a}$, which is deduced from the adsorbed mass using the molar weight and valency of the monomers (Figure S12). Calculating the surface charge density for formation of the PDADMAC/PSS multilayers, we obtain $\sigma_{\mathrm{a}}$ $=120.2 \pm 2.5 \mu \mathrm{C} / \mathrm{cm}^{2}$ for PDADMAC for $V_{\mathrm{GS}}=-400 \mathrm{mV}$ and $\sigma_{\mathrm{a}}=72.0 \pm 2.6 \mu \mathrm{C} / \mathrm{cm}^{2}$ for $V_{\mathrm{GS}}=+400 \mathrm{mV}$ and for PSS $\sigma_{\mathrm{a}}$ $=104.9 \pm 2.5 \mu \mathrm{C} / \mathrm{cm}^{2}$ for $V_{\mathrm{GS}}=-400 \mathrm{mV}$ and $\sigma_{\mathrm{a}}=62.9 \pm$ $2.6 \mu \mathrm{C} / \mathrm{cm}^{2}$ for $V_{\mathrm{GS}}=+400 \mathrm{mV}$. Subtracting the zerocapacitance data from the EG-FET response (green solid line) reveals the contribution of the induced surface charge density $\sigma_{\mathrm{i}}$ (purple dashed line).

\section{DISCUSSION}

The different nature of the SPR and EG-FET originates from the capacitive contributions $\left(\sigma_{\mathrm{i}}\right)$, yielding additional information about the charge distribution processes in PEMs. The SPR signal corresponds to mass uptake, attesting to the adsorption of long polymer chains in a fast process. Counterions surrounding the charged polymer backbone in the bulk solution are introduced into the PEM structure during surface adsorption. Oppositely charged PEMs achieve their electroneutrality by intrinsic charge compensation, resulting in expulsion of previously trapped counterions. This Donnan exclusion originates from the electroosmotic pressure of trapped counterions ${ }^{54}$ and from screening between chargelike polymers due to layer to layer charge neutralization. ${ }^{53,56}$ The resulting intralayer $\mathrm{K}^{+}$or $\mathrm{Cl}^{-}$flux can be described as slow non-Fickian diffusion ${ }^{50,57}$ to the solid-liquid interface, modifying the surface charge density $\sigma_{\mathrm{i}}{ }^{54}$ Hence, the potential in the PEM layer changes over time, and a corresponding electrical double layer forms at the polyelectrolyte interface, extending approximately $100 \mathrm{~nm}$ into the ion solution (Figure S16), ${ }^{35,58}$ leading to a modulation in the local electric field. The changes in charge distribution and ion concentration can also be described by the chemical potential by a thermodynamic approach (eq S19, Chapter S1C). Since our 
setup allows a direct measurement of mass and charge, we describe the equivalent by the more direct approach using a theoretical model for determination of the surface charge density utilizing a diffusion $\operatorname{model}^{58}$

$$
\sigma_{i}(t)=\sigma_{\mathrm{EDL}}\left(1-e^{\left(-t / t_{0}\right)}\right)+I_{\mathrm{f}} t
$$

where $I_{\mathrm{f}}$ is the Faraday current density at the gate. We measured a constant gate current $I_{\mathrm{GS}}$ (leakage current, $21 \mathrm{nA}$ ) during all experiments and deposition steps, thus indicating a negligible redox potential at the electrode (Figure S13). Therefore, we assume $I_{\mathrm{f}}$ to be close to zero. The standard FET equation for $I_{\mathrm{DS}}$ is

$$
I_{\mathrm{DS}}=\frac{W}{L} C_{i} \mu\left(V_{\mathrm{GS}}-V_{T}\right) V_{\mathrm{DS}}
$$

where $W$ is the channel width, $L$ the channel length, $C_{\mathrm{i}}$ the insulating layer capacitance, $\mu$ the charge mobility in the $\mathrm{rGO}$, and $V_{\mathrm{T}}$ the threshold voltage. Rewriting eq 4 as a function of the surface charge density ${ }^{59}$ and expressing the surface potential $\psi$ via the Debye-Hückel model (see eq 2), we obtain eq 5, which describes the modulation of the observed $I_{\mathrm{DS}}$ as a consequence of $\psi$ from $\sigma_{\mathrm{i}}$ due to ion diffusion and the depletion layer in the EDL

$$
I_{\mathrm{DS}}=I_{\mathrm{DS}, 0}-W d_{p} L \int_{V_{\mathrm{GS}}}^{V_{\mathrm{GS}}-V_{\mathrm{DS}}} \frac{\psi_{i}(V)}{\varepsilon \varepsilon_{0} \kappa} \mathrm{d} V \cdot\left(1-e^{\left(-t / t_{0}\right)}\right)
$$

where $d_{p}$ is the polyelectrolyte layer thickness. Equation 5 describes the modulation of the observed $I_{\mathrm{DS}}$ resulting from changes in the Fermi level of the rGO from the surface potential $\psi\left(V_{\mathrm{GS}}\right)$, which is determined by the voltage drop in the PEMs and the potential drop at the solid-electrolyte interface in proximity of the depletion layer (Figure 5). From eqs 2 and 5 it becomes clear that the corresponding potential drop $\psi_{i}$ results in a change of the observed EG-FET signal.

The EG-FET signal after PDADMAC deposition gradually decreased throughout rinsing with $\mathrm{KCl}$ solution and equilibrated over about $15 \mathrm{~min}$, while $\Delta$ SPR stabilized rapidly. This observation hints at the loss of approximately $5 \%$ of the loosely bound surface layer ${ }^{60}$ into which the majority of the ions previously diffused. The desorption results in a surface charge density alteration and triggers the corresponding EDL formation, leading to a minute SPR response but a distinct EG-FET response. Our observations originate from inherent material properties and corresponding ion interactions, leading to different charge densities at the surface. Therefore, rinsing with $\mathrm{KCl}$ after PSS deposition exhibits a less prominent but faster change to a stable current.

The applied electric field (from $V_{\mathrm{GS}}$ ) effects the diffusion, depending on the charge polarity of ions by either slowing or accelerating the diffusion flux. To evaluate the characteristics of the observed behavior, kinetics obtained from the EG-FET regarding $\sigma_{\mathrm{i}}$ were used for assessment of an arbitrary time ratio $\xi$, which is obtained from the time constant ratio of layer deposition and rinsing $\left(\xi=t_{\text {on }} / t_{\text {off }}\right)$ (Chapter S1c). Therefore, we assume that the obtained time ratio $\xi$ reflects a measure for ion affinity during diffusion, similar to diffusion-influenced transport in transmembrane channels. ${ }^{61} \xi=1.7 \pm 0.2$ for PDADMAC at $V_{\mathrm{GS}}=-400 \mathrm{mV}$ and $1.3 \pm 0.1$ for $+400 \mathrm{mV}$, while obtained $\xi$ values for PSS are $0.22 \pm 0.02$ at $-400 \mathrm{mV}$ and $0.32 \pm 0.04$ at $+400 \mathrm{mV}$. This is intuitive because negatively charged PEMs layers have stronger affinities toward

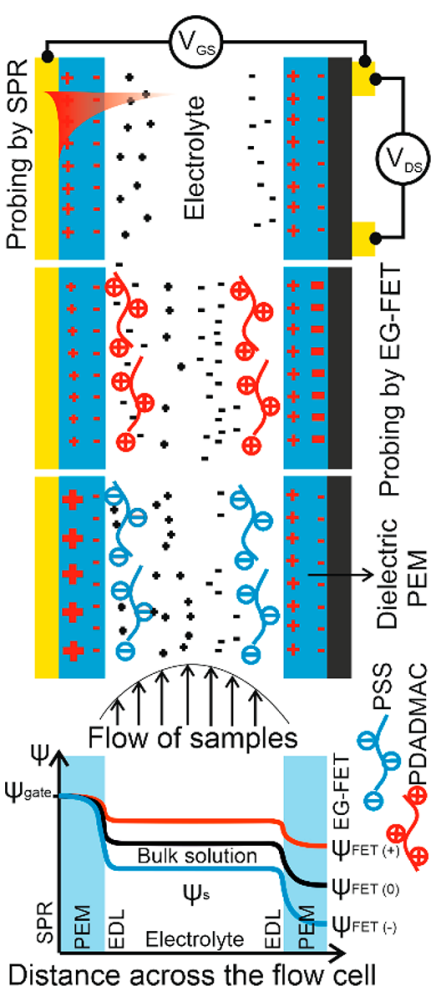

Figure 5. Schematics of the simultaneous readout of surface mass and charge density at the two interfaces of the combined SPR/EG-FET platform. Upper graph demonstrates initial conditions, followed by subsequent PDADMAC and PSS depositions under constant flow of polyelectrolyte solutions. Left side illustrates the SPR-Au-PEM surface, and right side represents the PEM-rGO-EG-FET. Surface charge density variations upon layer formation lead to the gate potential $(\Psi)$ drop across the fluidic channel that is shown below.

positively charged ions in the Helmholtz layer at positive applied $V_{\mathrm{GS}}$. The values of $\xi$ for PDADMAC and PSS PEMs are different as measured with the EG-FET. Most likely the differences of $\xi$ are related to the polarity of $V_{\mathrm{GS}}$, the corresponding Helmholtz double layer ion type, and the intrinsic charge of the PEMs. Furthermore, we speculate that the trapping of counterions is more pronounced in PSS layers due to ionic $\pi$-interactions, ${ }^{62}$ leading to slower ion diffusion in comparison to PDADMAC. Additionally, the $\pi-\pi$ interactions of the PSS layer itself could lead to a difference in interlayer ion diffusion flux.

\section{SUMMARY}

In summary, we herein present a powerful novel combinatorial sensing platform which provides new insights into real-time surface processes and enables direct measurement of surface charge density and mass obtained from electro/plasmonic signal transductions. We applied this platform to investigate PEM formation using a PDADMAC/PSS system. Due to the complementary sensing principles, the solid-liquid interface can be investigated from different perspectives, which is crucial as certain processes are beyond the scope of each individual tool. For instance, sole observation of the layer formation via SPR would suggest a completed material deposition within $60 \mathrm{~s}$, whereas the subsequent slower charge diffusion takes more than $15 \mathrm{~min}$ as observed by EG-FET. To the best of our knowledge, this is the first report of a SPR/EG-FET platform for simultaneous real-time monitoring under dynamic flow 
conditions, deconvoluting mass and charge contributions. On the basis of experimental observations, we derived a theoretical model to account for the evolution of surface charge density due to PEM adsorption and intralayer ion diffusion. The theory describes the time dependence of both mass uptake and charge distribution, elucidated from changes of the refractive index and surface potential at the solid-liquid interface. To this end, we combined the Debye-Hückel model and nonFickian diffusion theory to unravel intrinsic material processes. Correlation of optical and electronic read-outs allows for the discrimination of superimposed signals which originate from charged mass uptake and subsequent surface charge redistribution. We attribute each contribution to adsorbed and induced components by deconvoluting the superimposed yet time-correlated, EG-FET signal from the SPR data. By doing so, we can interpret an optical signal such that a direct comparison to an electronic signal is possible. Thus, our bifunctional sensor platform is a new tool to monitor surface events by simultaneously analyzing both the adsorbed mass and the intrinsic molecular charges at the same surface and under dynamic conditions. We hope these new insights can lead to a better understanding of intrinsic processes, aid in predicting material properties, and thus guide material and application development.

\section{ASSOCIATED CONTENT}

\section{(3) Supporting Information}

The Supporting Information is available free of charge at https://pubs.acs.org/doi/10.1021/jacs.9b11835.

Experimental details and fabrication procedures, additional EG-FET and SPR measurements, detailed theoretical framework, SPR/EG-FET setup, software, materials (PDF)

\section{AUTHOR INFORMATION}

\section{Corresponding Author}

Johannes Bintinger - Biosensor Technologies, Austrian Institute of Technology, 3430 Tulln, Austria; 이이이.org/0000-00026397-4254; Email: johannes.bintinger@ait.ac.at

\section{Authors}

Patrik Aspermair - Biosensor Technologies, Austrian Institute of Technology, 3430 Tulln, Austria; CEST Competence Center for Electrochemical Surface Technologies, 3430 Tulln, Austria; CNRS, Centrale Lille, ISEN, Universite Valenciennes, UMR 8520-IEMN, Universite de Lille, 59000 Lille, France; (D) orcid.org/0000-0003-1671-1328

Ulrich Ramach - CEST Competence Center for Electrochemical

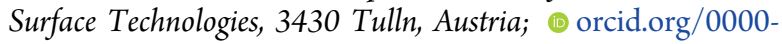
0002-1111-2994

Ciril Reiner-Rozman - Biosensor Technologies, Austrian Institute of Technology, 3430 Tulln, Austria; 이이.org/ 0000-0002-6476-2413

Stefan Fossati - Biosensor Technologies, Austrian Institute of Technology, 3430 Tulln, Austria; 이이이.org/0000-00021109-0035

Bernadette Lechner - Biosensor Technologies, Austrian Institute of Technology, 3430 Tulln, Austria; 이이.org/ 0000-0002-4949-5118

Sergio E. Moya - CIC biomaGUNE, 20014 San Sebastian, Spain; ○ orcid.org/0000-0002-7174-1960
Omar Azzaroni - Instituto de Investigaciones Fisicoquimicas Téricas y Aplicadas (INIFTA), Departamento de Quimica, Facultad de Ciencias Exactas, Universidad Nacional de La Plata-CONICET, 1900 La Plata, Argentina; (o orcid.org/ 0000-0002-5098-0612

Jakub Dostalek - Biosensor Technologies, Austrian Institute of

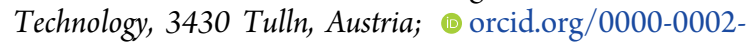
0431-2170

Sabine Szunerits - CNRS, Centrale Lille, ISEN, Universite Valenciennes, UMR 8520-IEMN, Universite de Lille, 59000 Lille, France; (1) orcid.org/0000-0002-1567-4943

Wolfgang Knoll - Biosensor Technologies, Austrian Institute of Technology, 3430 Tulln, Austria; CEST Competence Center for Electrochemical Surface Technologies, 3430 Tulln, Austria; - orcid.org/0000-0003-1543-4090

Complete contact information is available at:

https://pubs.acs.org/10.1021/jacs.9b11835

\section{Author Contributions}

${ }^{\perp}$ P.A. and U.R. contributed equally.

\section{Author Contributions}

This manuscript was written through contributions of all authors. All authors have given approval to the final version of the manuscript.

\section{Funding}

We gratefully acknowledge the financial support from the Austrian Research Promotion Agency (FFG; 870025) for this research. SF and JD are grateful for the support from the Austrian Research Promotion Agency (FFG; 861578, ERANET project PLABAN). This work was financially supported by the European Union's Horizon 2020 research and innovation program under grant agreement No 690836. General financial supports from the Centre National de la Recherche Scientifique (CNRS), the University of Lille, and the Hauts-de-France region are acknowledged.

\section{Notes}

The authors declare no competing financial interest.

\section{ACKNOWLEDGMENTS}

We thank Esteban Piccinini for valuable input and discussions and David Ebner for initial design input. Cover art by Ella Maru Studio.

\section{REFERENCES}

(1) Das, A.; Pisana, S.; Chakraborty, B.; Piscanec, S.; Saha, S. K.; Waghmare, U. V.; Novoselov, K. S.; Krishnamurthy, H. R.; Geim, A. K.; Ferrari, A. C.; Sood, A. K. Monitoring Dopants by Raman Scattering in an Electrochemically Top-Gated Graphene Transistor. Nat. Nanotechnol. 2008, 3 (4), 210-215.

(2) Kergoat, L.; Herlogsson, L.; Braga, D.; Piro, B.; Pham, M.-C.; Crispin, X.; Berggren, M.; Horowitz, G. A Water-Gate Organic FieldEffect Transistor. Adv. Mater. 2010, 22 (23), 2565-2569.

(3) Palazzo, G.; De Tullio, D.; Magliulo, M.; Mallardi, A.; Intranuovo, F.; Mulla, M. Y.; Favia, P.; Vikholm-Lundin, I.; Torsi, L. Detection Beyond Debye's Length with an Electrolyte-Gated Organic Field-Effect Transistor. Adv. Mater. 2015, 27 (5), 911-916.

(4) Casalini, S.; Leonardi, F.; Cramer, T.; Biscarini, F. Organic FieldEffect Transistor for Label-Free Dopamine Sensing. Org. Electron. 2013, 14 (1), 156-163.

(5) Ang, P. K.; Chen, W.; Wee, A. T. S.; Loh, K. P. Solution-Gated Epitaxial Graphene as PH Sensor. J. Am. Chem. Soc. 2008, 130 (44), 14392-14393. 
(6) Sailapu, S. K.; Macchia, E.; Merino-Jimenez, I.; Esquivel, J. P.; Sarcina, L.; Scamarcio, G.; Minteer, S. D.; Torsi, L.; Sabaté, N. Standalone Operation of an EGOFET for Ultra-Sensitive Detection of HIV. Biosens. Bioelectron. 2020, 156, 112103.

(7) Berto, M.; Diacci, C.; D’Agata, R.; Pinti, M.; Bianchini, E.; Lauro, M. D.; Casalini, S.; Cossarizza, A.; Berggren, M.; Simon, D.; Spoto, G.; Biscarini, F.; Bortolotti, C. A. EGOFET Peptide Aptasensor for Label-Free Detection of Inflammatory Cytokines in Complex Fluids. Adv. Biosyst. 2018, 2 (2), 1700072.

(8) Macchia, E.; Manoli, K.; Holzer, B.; Di Franco, C.; Ghittorelli, M.; Torricelli, F.; Alberga, D.; Mangiatordi, G. F.; Palazzo, G.; Scamarcio, G.; Torsi, L. Single-Molecule Detection with a MillimetreSized Transistor. Nat. Commun. 2018, 9 (1), 3223.

(9) Pappa, A.-M.; Parlak, O.; Scheiblin, G.; Mailley, P.; Salleo, A.; Owens, R. M. Organic Electronics for Point-of-Care Metabolite Monitoring. Trends Biotechnol. 2018, 36 (1), 45-59.

(10) Homola, J. Surface Plasmon Resonance Sensors for Detection of Chemical and Biological Species. Chem. Rev. 2008, 108 (2), 462493.

(11) Liedberg, B.; Nylander, C.; Lunström, I. Surface Plasmon Resonance for Gas Detection and Biosensing. Sens. Actuators 1983, 4, 299-304.

(12) Olaru, A.; Bala, C.; Jaffrezic-Renault, N.; Aboul-Enein, H. Y. Surface Plasmon Resonance (SPR) Biosensors in Pharmaceutical Analysis. Crit. Rev. Anal. Chem. 2015, 45 (2), 97-105.

(13) Homola, J. Present and Future of Surface Plasmon Resonance Biosensors. Anal. Bioanal. Chem. 2003, 377 (3), 528-539.

(14) Englebienne, P.; Hoonacker, A. V.; Verhas, M. Surface Plasmon Resonance: Principles, Methods and Applications in Biomedical Sciences. Spectroscopy 2003, 17 (2-3), 255-273.

(15) Piliarik, M.; Vaisocherová, H.; Homola, J. Surface Plasmon Resonance Biosensing. In Biosensors and Biodetection; Rasooly, A., Herold, K. E., Eds.; Methods in Molecular BiologyTM; Humana Press: Totowa, NJ, 2009; pp 65-88. DOI: 10.1007/978-1-60327567-5_5.

(16) Bouffartigues, E.; Leh, H.; Anger-Leroy, M.; Rimsky, S.; Buckle, M. Rapid Coupling of Surface Plasmon Resonance (SPR and SPRi) and ProteinChipTM Based Mass Spectrometry for the Identification of Proteins in Nucleoprotein Interactions. Nucleic Acids Res. 2007, 35 (6), e39-e39.

(17) Zhang, X.; Young, M. A.; Lyandres, O.; Van Duyne, R. P. Rapid Detection of an Anthrax Biomarker by Surface-Enhanced Raman Spectroscopy. J. Am. Chem. Soc. 2005, 127 (12), 4484-4489.

(18) Sergelen, K.; Fossati, S.; Turupcu, A.; Oostenbrink, C.; Liedberg, B.; Knoll, W.; Dostálek, J. Plasmon Field-Enhanced Fluorescence Energy Transfer for Hairpin Aptamer Assay Readout. ACS Sens. 2017, 2 (7), 916-923.

(19) Rodrigo, D.; Tittl, A.; Ait-Bouziad, N.; John-Herpin, A.; Limaj, O.; Kelly, C.; Yoo, D.; Wittenberg, N. J.; Oh, S.-H.; Lashuel, H. A.; Altug, H. Resolving Molecule-Specific Information in Dynamic Lipid Membrane Processes with Multi-Resonant Infrared Metasurfaces. Nat. Commun. 2018, 9 (1), 1-9.

(20) Mulla, M. Y.; Tuccori, E.; Magliulo, M.; Lattanzi, G.; Palazzo, G.; Persaud, K.; Torsi, L. Capacitance-Modulated Transistor Detects Odorant Binding Protein Chiral Interactions. Nat. Commun. 2015, 6, 6010.

(21) Panzer, M. J.; Frisbie, C. D. Exploiting Ionic Coupling in Electronic Devices: Electrolyte-Gated Organic Field-Effect Transistors. Adv. Mater. 2008, 20 (16), 3177-3180.

(22) Barbaro, M.; Bonfiglio, A.; Raffo, L. A Charge-Modulated FET for Detection of Biomolecular Processes: Conception, Modeling, and Simulation. IEEE Trans. Electron Devices 2006, 53 (1), 158-166.

(23) Cramer, T.; Kyndiah, A.; Murgia, M.; Leonardi, F.; Casalini, S.; Biscarini, F. Double Layer Capacitance Measured by Organic Field Effect Transistor Operated in Water. Appl. Phys. Lett. 2012, 100 (14), 143302 .

(24) Ohno, Y.; Maehashi, K.; Yamashiro, Y.; Matsumoto, K. Electrolyte-Gated Graphene Field-Effect Transistors for Detecting PH and Protein Adsorption. Nano Lett. 2009, 9 (9), 3318-3322.
(25) Torsi, L.; Magliulo, M.; Manoli, K.; Palazzo, G. Organic FieldEffect Transistor Sensors: A Tutorial Review. Chem. Soc. Rev. 2013, 42 (22), 8612-8628.

(26) Tibaldi, A.; Fillaud, L.; Anquetin, G.; Woytasik, M.; Zrig, S.; Piro, B.; Mattana, G.; Noël, V. Electrolyte-Gated Organic Field-Effect Transistors (EGOFETs) as Complementary Tools to Electrochemistry for the Study of Surface Processes. Electrochem. Commun. 2019, 98, 43-46.

(27) Nakatsuka, N.; Yang, K.-A.; Abendroth, J. M.; Cheung, K. M.; Xu, X.; Yang, H.; Zhao, C.; Zhu, B.; Rim, Y. S.; Yang, Y.; Weiss, P. S.; Stojanović, M. N.; Andrews, A. M. Aptamer-Field-Effect Transistors Overcome Debye Length Limitations for Small-Molecule Sensing. Science 2018, 362 (6412), 319-324.

(28) Goda, T.; Maeda, Y.; Miyahara, Y. Simultaneous Monitoring of Protein Adsorption Kinetics Using a Quartz Crystal Microbalance and Field-Effect Transistor Integrated Device. Anal. Chem. 2012, 84 (17), $7308-7314$

(29) Okuda, S.; Ono, T.; Kanai, Y.; Ikuta, T.; Shimatani, M.; Ogawa, S.; Maehashi, K.; Inoue, K.; Matsumoto, K. Graphene Surface Acoustic Wave Sensor for Simultaneous Detection of Charge and Mass. ACS Sens. 2018, 3 (1), 200-204.

(30) Kojori, H. S.; Ji, Y.; Paik, Y.; Braunschweig, A. B.; Kim, S. J. Monitoring Interfacial Lectin Binding with Nanomolar Sensitivity Using a Plasmon Field Effect Transistor. Nanoscale 2016, 8 (39), 17357-17364.

(31) Decher, G.; Eckle, M.; Schmitt, J.; Struth, B. Layer-by-Layer Assembled Multicomposite Films. Curr. Opin. Colloid Interface Sci. 1998, 3 (1), 32-39.

(32) Dubas, S. T.; Schlenoff, J. B. Factors Controlling the Growth of Polyelectrolyte Multilayers. Macromolecules 1999, 32 (24), 81538160.

(33) Ai, H.; Jones, S. A.; Lvov, Y. M. Biomedical Applications of Electrostatic Layer-by-Layer Nano-Assembly of Polymers, Enzymes, and Nanoparticles. Cell Biochem. Biophys. 2003, 39 (1), 23.

(34) Piccinini, E.; Bliem, C.; Reiner-Rozman, C.; Battaglini, F.; Azzaroni, O.; Knoll, W. Enzyme-Polyelectrolyte Multilayer Assemblies on Reduced Graphene Oxide Field-Effect Transistors for Biosensing Applications. Biosens. Bioelectron. 2017, 92, 661-667.

(35) Piccinini, E.; Alberti, S.; Longo, G. S.; Berninger, T.; Breu, J.; Dostalek, J.; Azzaroni, O.; Knoll, W. Pushing the Boundaries of Interfacial Sensitivity in Graphene FET Sensors: Polyelectrolyte Multilayers Strongly Increase the Debye Screening Length. J. Phys. Chem. C 2018, 122 (18), 10181-10188.

(36) Michel, M.; Taylor, A.; Sekol, R.; Podsiadlo, P.; Ho, P.; Kotov, N.; Thompson, L. High-Performance Nanostructured Membrane Electrode Assemblies for Fuel Cells Made by Layer-By-Layer Assembly of Carbon Nanocolloids. Adv. Mater. 2007, 19 (22), 3859-3864.

(37) Wu, F.; Li, J.; Su, Y.; Wang, J.; Yang, W.; Li, N.; Chen, L.; Chen, S.; Chen, R.; Bao, L. Layer-by-Layer Assembled Architecture of Polyelectrolyte Multilayers and Graphene Sheets on Hollow Carbon Spheres/Sulfur Composite for High-Performance Lithium-Sulfur Batteries. Nano Lett. 2016, 16 (9), 5488-5494.

(38) Ariga, K.; Lvov, Y. M.; Kawakami, K.; Ji, Q.; Hill, J. P. Layer-byLayer Self-Assembled Shells for Drug Delivery. Adv. Drug Delivery Rev. 2011, 63 (9), 762-771.

(39) Gu, J.-E.; Lee, S.; Stafford, C. M.; Lee, J. S.; Choi, W.; Kim, B.Y.; Baek, K.-Y.; Chan, E. P.; Chung, J. Y.; Bang, J.; Lee, J.-H. Molecular Layer-by-Layer Assembled Thin-Film Composite Membranes for Water Desalination. Adv. Mater. 2013, 25 (34), 47784782.

(40) Richardson, J. J.; Cui, J.; Björnmalm, M.; Braunger, J. A.; Ejima, H.; Caruso, F. Innovation in Layer-by-Layer Assembly. Chem. Rev. 2016, 116 (23), 14828-14867.

(41) Richardson, J. J.; Bjornmalm, M.; Caruso, F. TechnologyDriven Layer-by-Layer Assembly of Nanofilms. Science 2015, 348 (6233), aaa2491-aaa2491.

(42) Aspnes, D. E. Local-field Effects and Effective-medium Theory: A Microscopic Perspective. Am. J. Phys. 1982, 50 (8), 704-709. 
(43) Ogieglo, W.; Wormeester, H.; Eichhorn, K.-J.; Wessling, M.; Benes, N. E. In Situ Ellipsometry Studies on Swelling of Thin Polymer Films: A Review. Prog. Polym. Sci. 2015, 42, 42-78.

(44) Piccinini, E.; Bliem, C.; Giussi, J. M.; Knoll, W.; Azzaroni, O. Reversible Switching of the Dirac Point in Graphene Field-Effect Transistors Functionalized with Responsive Polymer Brushes. Langmuir 2019, 35, 8038.

(45) Tang, K.; Besseling, N. A. M. Formation of Polyelectrolyte Multilayers: Ionic Strengths and Growth Regimes. Soft Matter 2016, 12 (4), 1032-1040.

(46) De Feijter, J. A.; Benjamins, J.; Veer, F. A. Ellipsometry as a Tool to Study the Adsorption Behavior of Synthetic and Biopolymers at the Air-Water Interface. Biopolymers 1978, 17 (7), 1759-1772.

(47) Stenberg, E.; Persson, B.; Roos, H.; Urbaniczky, C. Quantitative Determination of Surface Concentration of Protein with Surface Plasmon Resonance Using Radiolabeled Proteins. J. Colloid Interface Sci. 1991, 143 (2), 513-526.

(48) Perlmann, G. E.; Longsworth, L. G. The Specific Refractive Increment of Some Purified Proteins. J. Am. Chem. Soc. 1948, 70 (8), 2719-2724.

(49) Wang, Y. Y.; Burke, P. J. Polyelectrolyte Multilayer Electrostatic Gating of Graphene Field-Effect Transistors. Nano Res. 2014, 7 (11), 1650-1658.

(50) Donath, E.; Vardanyan, I.; Meyer, S.; Murray, R. A.; Moya, S. E.; Navoyan, Z.; Arakelyan, V. A Typical Diffusion Monitored by Flow Cytometry: Slow Diffusion of Small Molecules in Polyelectrolyte Multilayers. Nanoscale 2018, 10 (2), 765-772.

(51) Wong, J. E.; Zastrow, H.; Jaeger, W.; von Klitzing, R. Specific Ion versus Electrostatic Effects on the Construction of Polyelectrolyte Multilayers $\dagger$. Langmuir 2009, 25 (24), 14061-14070.

(52) Ko, Y. H.; Kim, Y. H.; Park, J.; Nam, K. T.; Park, J. H.; Yoo, P. J. Electric-Field-Assisted Layer-by-Layer Assembly of Weakly Charged Polyelectrolyte Multilayers. Macromolecules 2011, 44 (8), 2866-2872.

(53) Kim, Y.-T.; Lee, S.; Park, S.; Lee, C. Y. Graphene Chemiresistors Modified with Functionalized Triphenylene for Highly Sensitive and Selective Detection of Dimethyl Methylphosphonate. RSC Adv. 2019, 9 (58), 33976-33980.

(54) Sui, Z.; Schlenoff, J. B. Phase Separations in PH-Responsive Polyelectrolyte Multilayers: Charge Extrusion versus Charge Expulsion. Langmuir 2004, 20 (14), 6026-6031.

(55) Kłos, J. S. Dendritic Polyelectrolytes Revisited through the Poisson-Boltzmann-Flory Theory and the Debye-Hückel Approximation. Phys. Chem. Chem. Phys. 2018, 20 (4), 2693-2703.

(56) Dai, J.; Balachandra, A. M.; Lee, J. I.; Bruening, M. L. Controlling Ion Transport through Multilayer Polyelectrolyte Membranes by Derivatization with Photolabile Functional Groups. Macromolecules 2002, 35 (8), 3164-3170.

(57) Song, L.; Sun, W.; Gao, J. Time Dependent Chloride Diffusion Coefficient in Concrete. J. Wuhan Univ. Technol., Mater. Sci. Ed. 2013, 28 (2), 314-319.

(58) Tybrandt, K.; Zozoulenko, I. V.; Berggren, M. Chemical Potential-Electric Double Layer Coupling in Conjugated PolymerPolyelectrolyte Blends. Sci. Adv. 2017, 3 (12), eaao3659.

(59) Shirinskaya, A.; Horowitz, G.; Rivnay, J.; Malliaras, G.; Bonnassieux, Y. Numerical Modeling of an Organic Electrochemical Transistor. Biosensors 2018, 8 (4), 103.

(60) Iturri Ramos, J. J.; Stahl, S.; Richter, R. P.; Moya, S. E. Water Content and Buildup of Poly(Diallyldimethylammonium Chloride)/ Poly(Sodium 4-Styrenesulfonate) and Poly(Allylamine Hydrochloride)/Poly(Sodium 4-Styrenesulfonate) Polyelectrolyte Multilayers Studied by an in Situ Combination of a Quartz Crystal Microbalance with Dissipation Monitoring and Spectroscopic Ellipsometry. Macromolecules 2010, 43 (21), 9063-9070.

(61) Zhou, H.-X. Diffusion-Influenced Transport of Ions across a Transmembrane Channel with an Internal Binding Site. J. Phys. Chem. Lett. 2010, 1 (13), 1973-1976.

(62) Schottel, B. L.; Chifotides, H. T.; Dunbar, K. R. Anion- $\pi$ Interactions. Chem. Soc. Rev. 2008, 37 (1), 68-83. 